Supplementary Information

\title{
Emergence of Metallic Conduction and Cobalt(II)-based Single- Molecule Magnetism in the Same Temperature Range
}

Yongbing Shen, ${ }^{* a}$ Hiroshi Ito, ${ }^{*}$ b Haitao Zhang, ${ }^{\mathrm{c}}$ Hideki Yamochi, ${ }^{\mathrm{d}, \mathrm{e}}$ Goulven Cosquer, ${ }^{\mathrm{f}}$ Carmen Herrmann, ${ }^{\mathrm{c}}$ Toshiaki Ina, ${ }^{\mathrm{g}}$ Shinji K. Yoshina, ${ }^{\mathrm{b}}$ Brian K. Breedlove, ${ }^{\mathrm{a}}$ Akihiro Otsuka, ${ }^{\mathrm{d}}$ e Manabu Ishikawa, ${ }^{\mathrm{d}}$ Takefumi Yoshida, ${ }^{\mathrm{a}}$ and Masahiro Yamashita, ${ }^{*} \mathrm{a}, \mathrm{h}$

${ }^{a}$ Department of Chemistry, Graduate School of Science, Tohoku University, Sendai, Japan

${ }^{\mathrm{b}}$ Department of Applied Physics, Nagoya University, Chikusa-ku, Nagoya 464-603, Japan

${ }^{\mathrm{c}}$ Institute of Inorganic and Applied Chemistry, University of Hamburg, Martin-Luther-King-

Platz 6, 20146 Hamburg, Germany

${ }^{\mathrm{d}}$ Division of Chemistry, Graduate School of Science, Kyoto University, Sakyo-ku, Kyoto 606 8502, Japan

${ }^{\mathrm{e}}$ Research Center for Low Temperature and Materials Sciences, Kyoto University, Sakyo-ku, Kyoto 606-8501, Japan

${ }^{\mathrm{f}}$ Research Group of Solid Material Chemistry, Graduate School of Science, Hiroshima University 1-3-1 Kagamiyama, Higashihiroshima, Hiroshima, 739-8526, Japan

g Spectroscopy and Imaging Division, Japan Synchrotron Radiation Research Institute, 1-1-1 Kouto, Sayo, Sayou-gun, Hyogo 679-5198, Japan

${ }^{\mathrm{h}}$ School of Materials Science and Engineering, Nankai University, Tianjin 300350, China 


\section{Experimental Methods}

General information: $\mathrm{H}_{2} \mathrm{pdms}, \mathrm{CoCl}_{2}$, and organic solvents were commercially purchased and used without any further purification. Neutral BO was obtained as the by-product in the synthesis of ethylenedioxytetrathiafulvalene ${ }^{1}$ and purified by recrystallization from cyclohexane. $\left(\mathrm{HNEt}_{3}\right)_{2}\left[\mathrm{Co}(\mathrm{pdms})_{2}\right]$ was synthesized by following reported procedures ${ }^{2}$ and recrystallized two times from acetonitrile-diethyl ether before use.

Preparation of $\beta^{\prime \prime}-(\mathrm{BEDO}-\mathrm{TTF})_{3}\left[\mathrm{Co}(\mathrm{pdms})_{2}\right] \cdot(\mathrm{MeCN})\left(\mathrm{H}_{2} \mathrm{O}\right)_{2}$ : Neutral BO (15 mg) and $\left(\mathrm{HNEt}_{3}\right)_{2}\left[\mathrm{Co}(\mathrm{pdms})_{2}\right](100 \mathrm{mg})$ were dissolved in a mixture of acetonitrile $(18 \mathrm{~mL})$, and two drops of distilled water, and the resulting solution was placed in an $\mathrm{H}$-shaped cell under $\mathrm{N}_{2}$ gas atmosphere. The dc current $(0.7 \mu \mathrm{A})$ was then applied. Large amount of black thin plate-like crystals with typical size of $0.5 \times 0.1 \times 0.02 \mathrm{~mm}^{3}$ (Figure $\mathrm{S} 6$ ) grew on the anode over 18 days. The crystals were collected, washed with a small amount of acetonitrile and dried in air. Yield: $\sim 60 \%$ (based on neutral BEDO-TTF). Elemental analysis: $\mathrm{C}_{48} \mathrm{H}_{51} \mathrm{~N}_{5} \mathrm{O}_{22} \mathrm{~S}_{16} \mathrm{Co}_{1}$ Calcd. (\%): $\mathrm{C}$, 35.54; H, 3.17; N, 4.32, S, 31.63. Found (\%): C, 35.71; H, 3.54; N, 3.98; S, 31.46.

Physical characterization: Single-crystal X-ray crystallographic data were collected at $120 \mathrm{~K}$ on a Rigaku Saturn70 CCD Diffractometer (Rigaku, Tokyo, Japan) with graphitemonochromated Mo $\mathrm{K} \alpha$ radiation $(\lambda=0.71073 \AA)$ produced by a VariMax microfocus X-ray rotating anode source. Data processing was performed using the Crystal-Clear crystallographic software package. ${ }^{3}$ The structures were solved by using direct methods included in SIR-92, ${ }^{4}$ and refinement was carried out using SHELXL-2013. ${ }^{5}$ The non-H atoms were refined anisotropically using weighted full-matrix least squares, and $\mathrm{H}$ atoms attached to the $\mathrm{C}$ atoms were positioned using idealized geometries and refined using a riding model. Elemental analysis was performed at the Research and Analytical Centre for Giant Molecules, Tohoku University.

UV-Vis-IR spectrum were acquired in the solid-state as $\mathrm{KBr}$ pellets on a Shimadzu UV3100pc (Shimadzu, Kyoto, Japan) and a FT-IR-6200YMS infrared spectrometer (JASCO, Tokyo, Japan) at room temperature. Raman spectroscopy was performed on single crystals using a Micro Laser Raman Spectrometer LabRam H-800 at an excitation of $532 \mathrm{~nm}$ at room temperature.

Magnetic susceptibility measurements were conducted on polycrystalline samples using a Quantum Design SQUID magnetometer MPMS-7L. Diamagnetic corrections were estimated from Pascal's constants $\left(-801 \times 10^{-6} \mathrm{~cm}^{3} \mathrm{~mol}^{-1}\right){ }^{6} A C$ measurements were performed in the frequency range of $0.1-1000 \mathrm{~Hz}$. Temperature dependences of electrical conductivity $(\sigma)$ were determined with a four-probe method. 
Electronic structure calculations: The PBC (Periodic boundary conditions) DFT were performed with the VASP package within the Perdew-Burke-Ernzerhof (PBE) exchangecorrelation functional. PAW pseudopotentials (Name/the number of valence electron: Co_sv/17, S_h/6, O/6, N/5, C/4, H/1) and plane-wave basis sets with cutoff energies of $800 \mathrm{eV}$ for wavefunctions were used. A $4 \times 4 \times 2$ Monkhorst-Pack k-mesh was employed for BO3. 


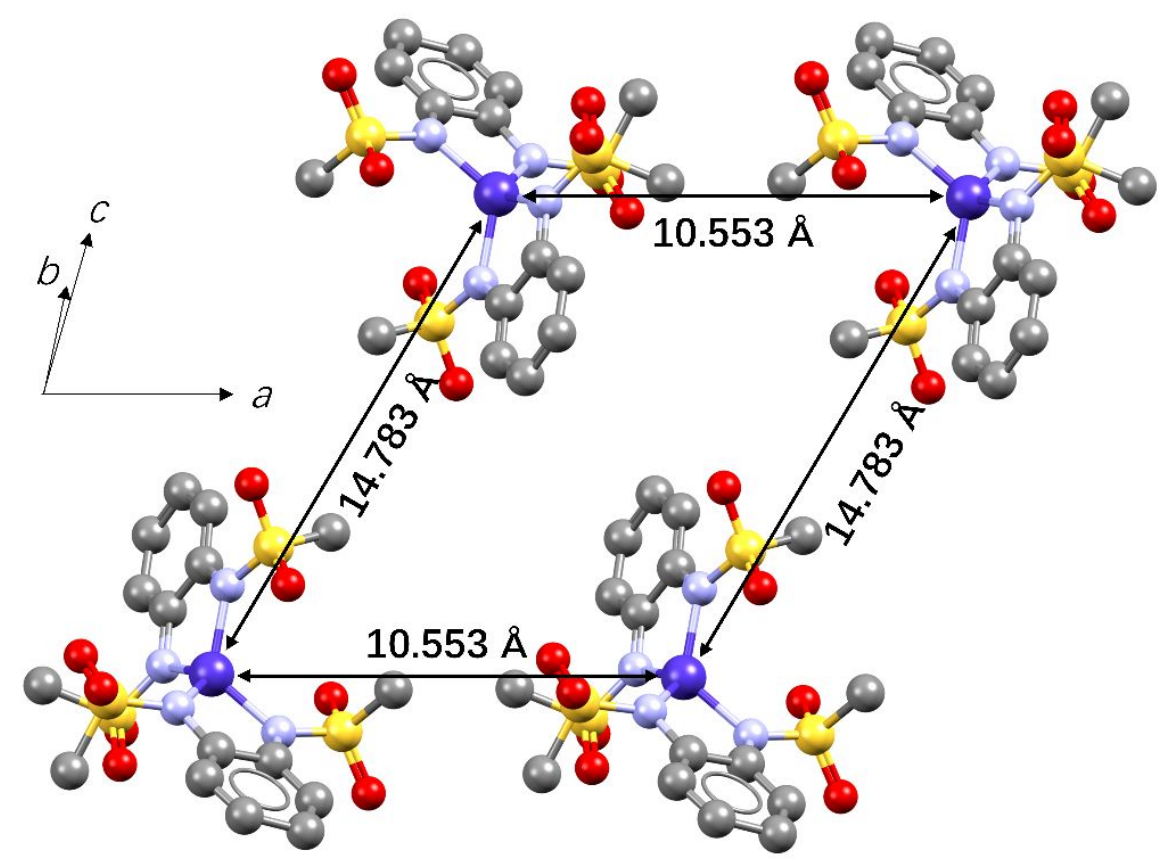

Figure S1. The arrangement of $\mathrm{Co}(\mathrm{pdms})_{2}$ in magnetic layer and the distance $(\AA)$ between Co ions in SMM layer. 

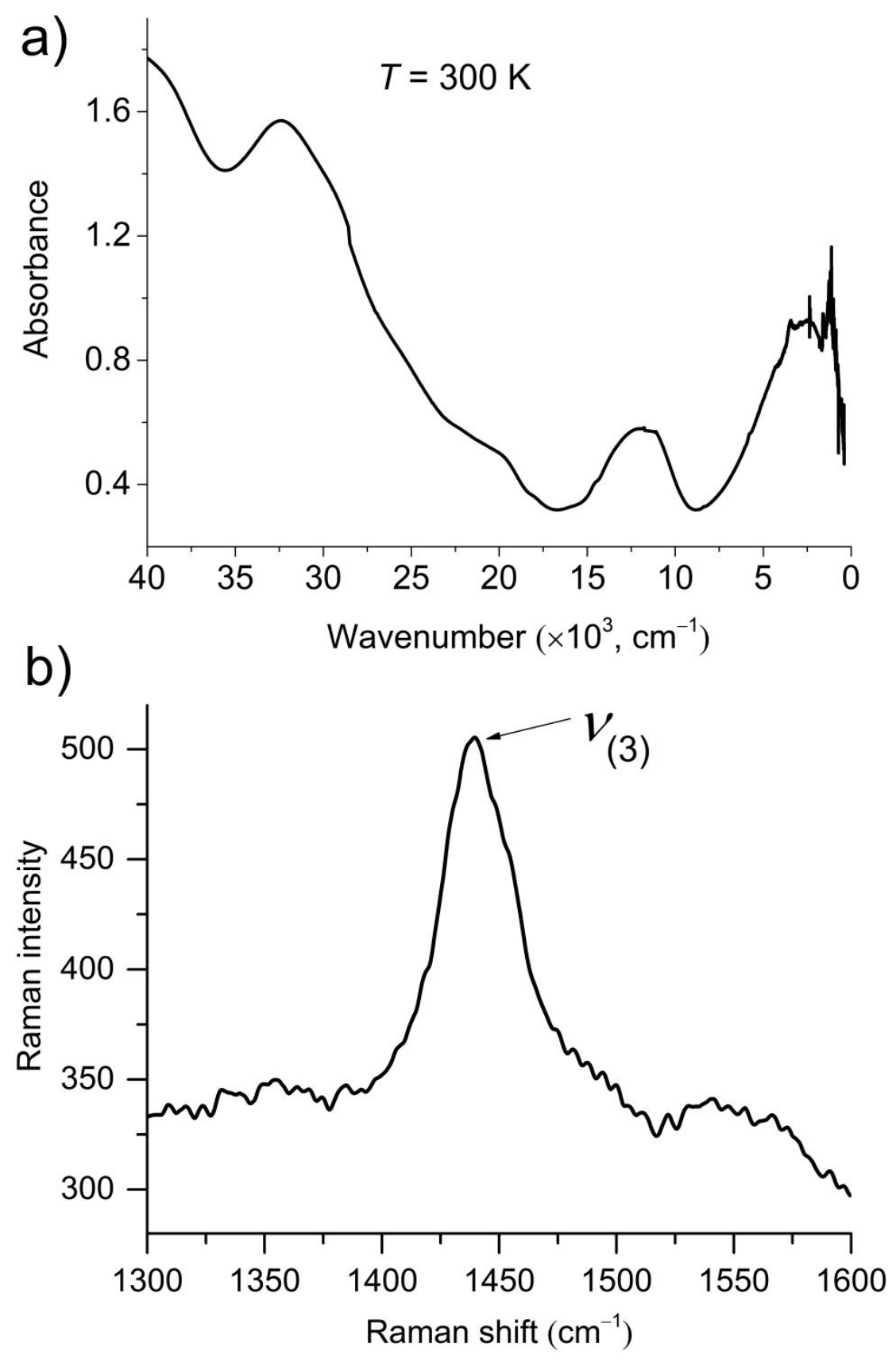

Figure S2. Spectroscopic properties of BO3. a) Optical absorbance in $550-40000 \mathrm{~cm}^{-1}$ at 300 K. b) Raman spectrum in $1300-1600 \mathrm{~cm}^{-1}$ at $300 \mathrm{~K}$. 


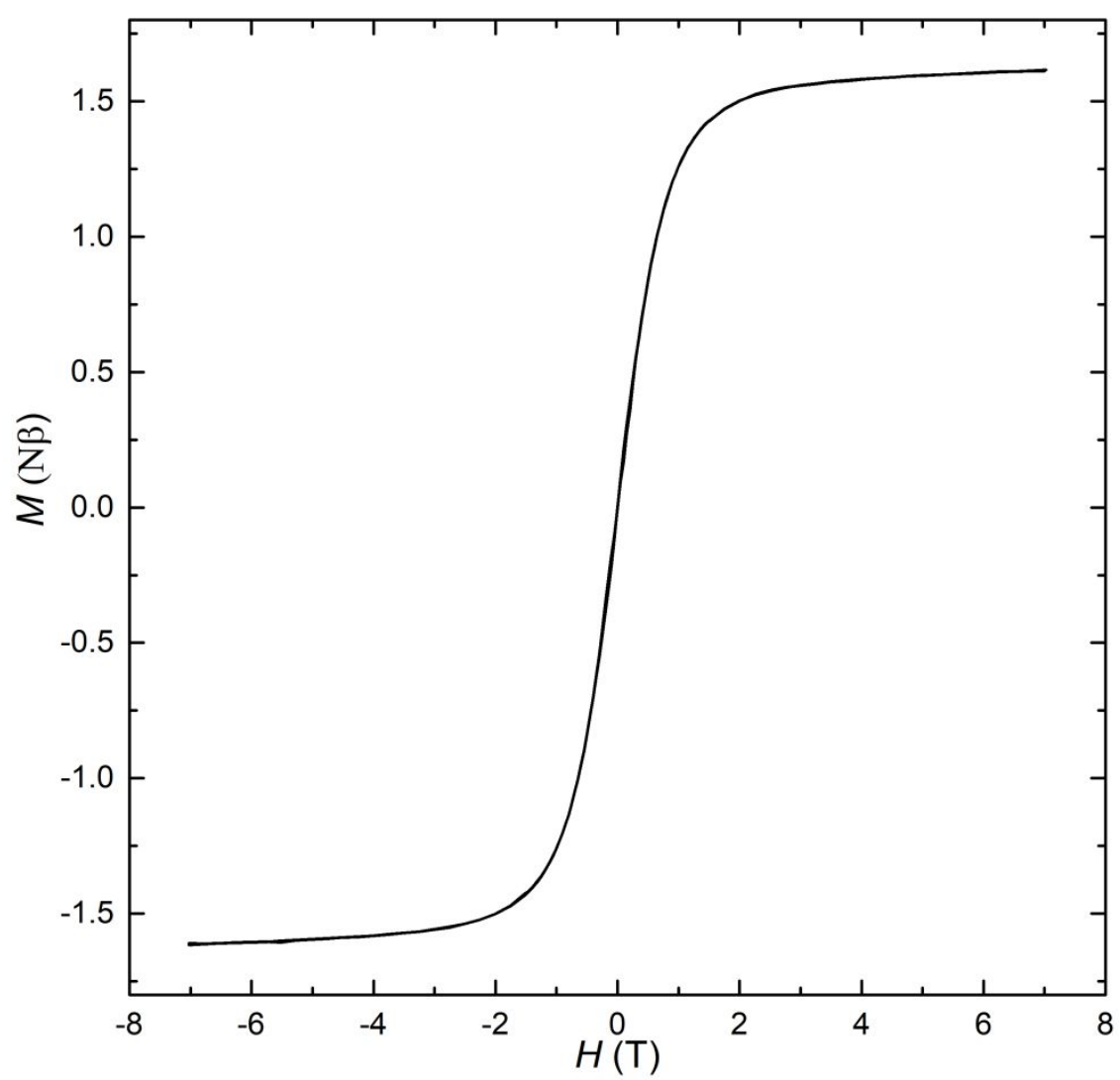

Figure S3. The polycrystal-based magnetic field (H) dependence of magnetization (M) at 1.85 $\mathrm{K}$ in -7 to $7 \mathrm{~T}$. 


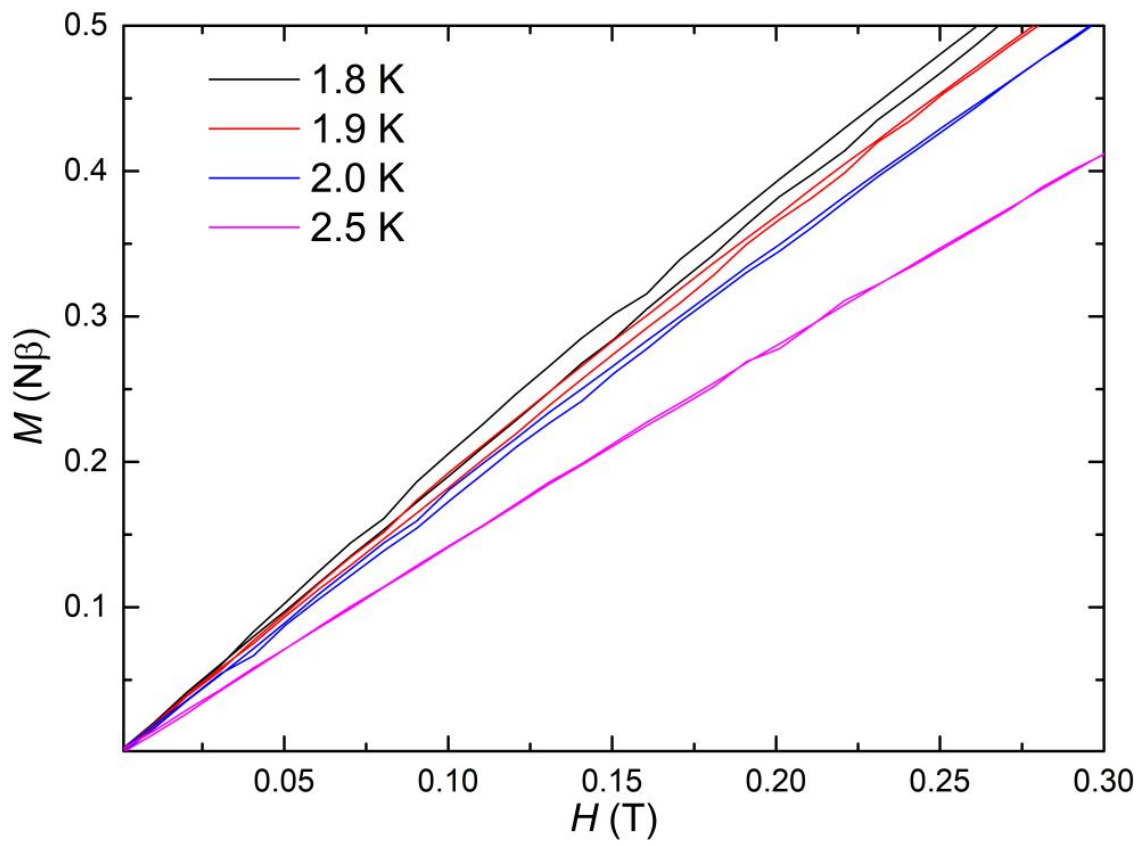

Figure S4. The magnetic field $(\mathrm{H})$ dependence of magnetization $(\mathrm{M})$ in $0-0.3 \mathrm{~T}$ at $1.8,1.9,2.0$ and $2.5 \mathrm{~K}$. 

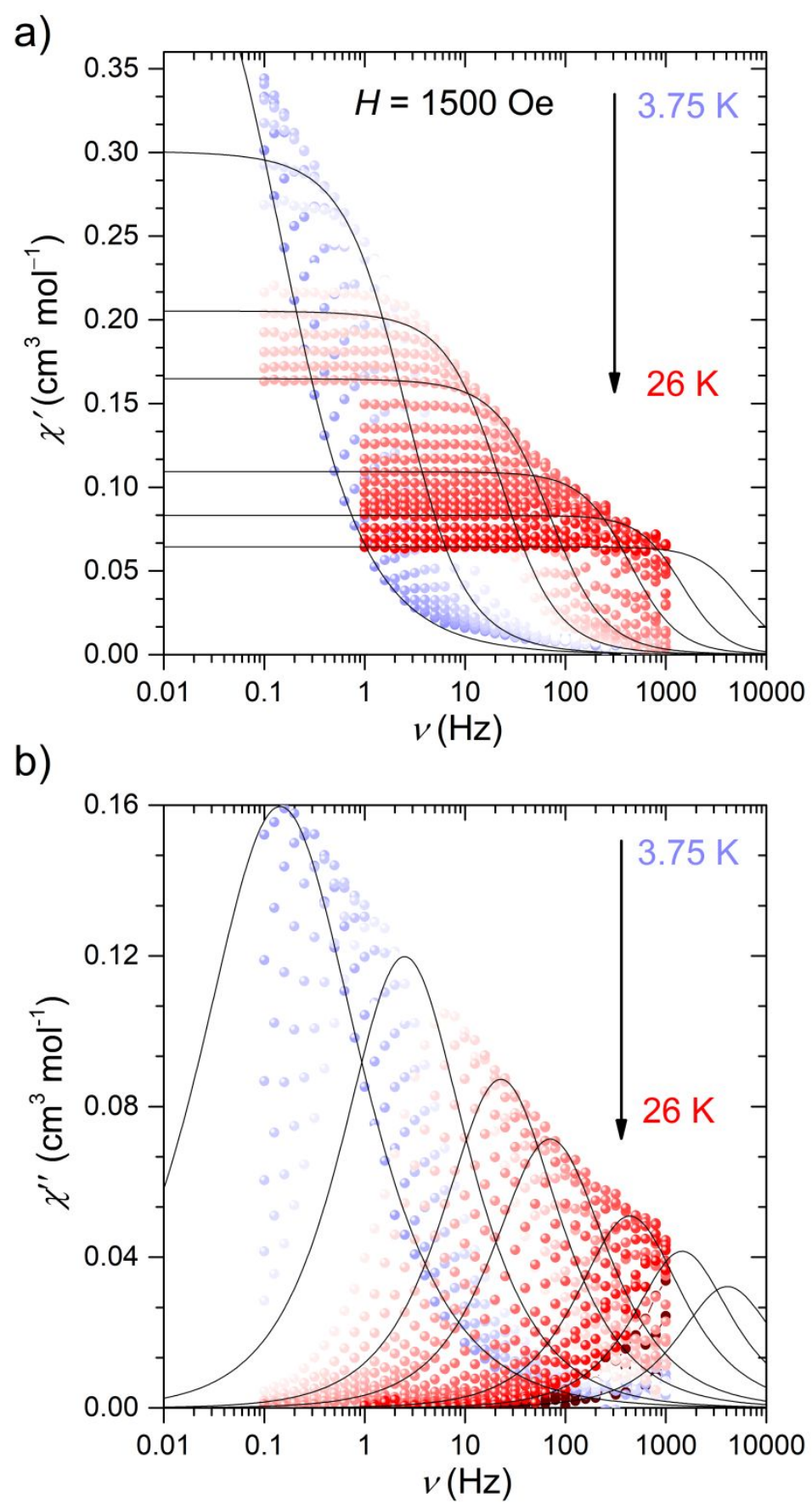

Figure S5. $A C$ magnetic susceptibility of BO3. a) Frequency dependence of in-phase of magnetic susceptibility $\left(\chi^{\prime}\right)$ in $3.75-26 \mathrm{~K}$ at 1500 Oe field. b) Frequency dependence of out-ofphase of magnetic susceptibility $\left(\chi^{\prime \prime}\right)$ in $3.75-26 \mathrm{~K}$ at 1500 Oe field. The black curves represent the best fit. 


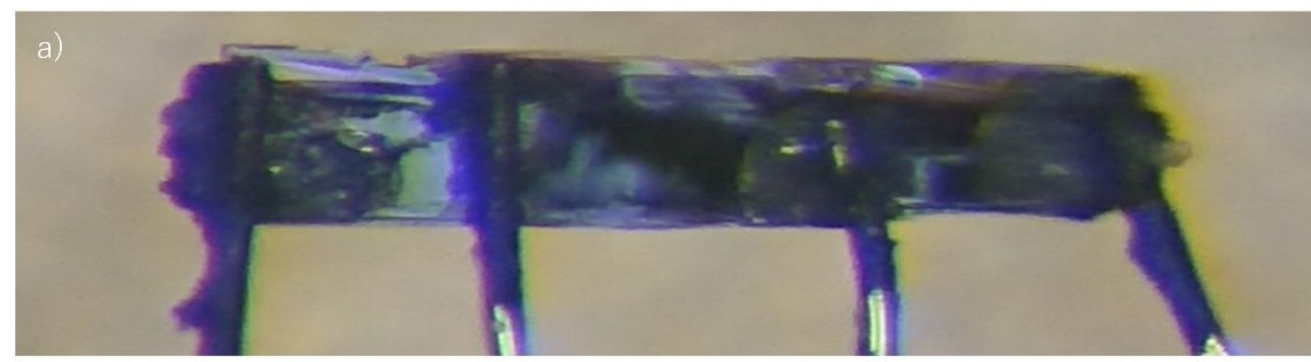

$$
\begin{aligned}
& \text { Dimensions: } \\
& \text { - Length }=0.5 \mathrm{~mm} \\
& \text { - Width }=0.14 \mathrm{~mm} \\
& \text { - Height } \sim 0.02 \mathrm{~mm}
\end{aligned}
$$

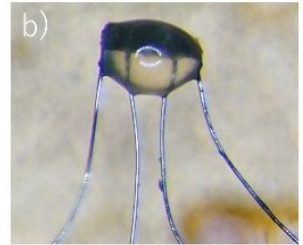

Figure S6. a) The single-crystal for electrical conductivity measurement with four-probe method. b) Due to the fragile quality (very thin), the crystals were coated by grease to avoid breaking during the cooling. 


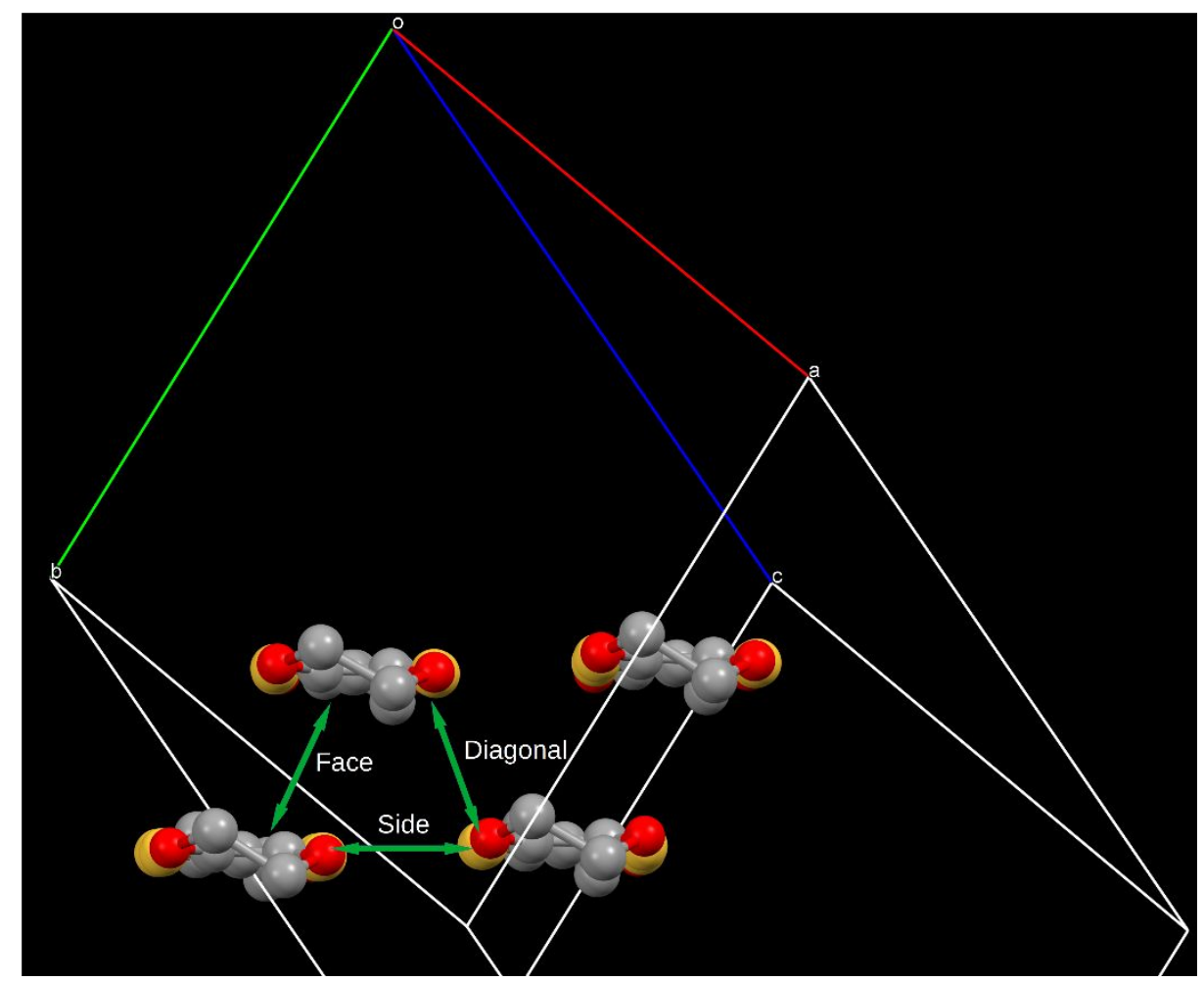

Figure S7. The face, diagonal and side kinds of interactions between three BEDO-TTF molecules in $\mathbf{B O 3}$ unit cell. 


\section{References}

1. Iyoda, M.; Kuwatani, Y.; Ogura, E.; Hara, K.; Suzuki, H.; Takano, T.; Takeda, K.; Takano, J.; Ugawa, K.; Yoshida, M.; Matsuyama, H.; Nishikawa, H.; Ikemoto, I.; Kato, T.; Yoneyama, N.; Nishijo, J.; Miyazaki, A.; Enoki, T., Syntheses, structure and conducting properties of halogenated ethylenedioxytetrathiafulvalenes. Heterocycles 2001, 54 (2), 833-+.

2. Rechkemmer, Y.; Breitgoff, F. D.; van der Meer, M.; Atanasov, M.; Hakl, M.; Orlita, M.; Neugebauer, P.; Neese, F.; Sarkar, B.; van Slageren, J., A four-coordinate cobalt(II) single-ion magnet with coercivity and a very high energy barrier. Nat.Commun. 2016, 7.

3. CrystalClear-SM 1.4.0 SP1, C., Rigaku.

4. Altomare, A.; Burla, M. C.; Camalli, M.; Cascarano, G. L.; Giacovazzo, C.; Guagliardi, A.; Moliterni, A. G. G.; Polidori, G.; Spagna, R., SIR97: a new tool for crystal structure determination and refinement. J. Appl. Crystallogr. 1999, 32, 115-119.

5. Sheldrick, G. M., A short history of SHELX. Acta. Crystallographica a-Foundation and Advances. 2008, 64, 112-122.

6. Bain, G. A.; Berry, J. F., Diamagnetic corrections and Pascal's constants. J. Chem. Educ. 2008, 85 (4), 532-536. 